\title{
Photodynamic activity of pyropheophorbide methyl ester and pyropheophorbide a in dimethylformamide solution
}

\author{
Saleh Al-Omari and Ahmad Ali \\ Department of Physics, The Hashemite University, Zarqa 13115, Jordan
}

\begin{abstract}
Comparative spectroscopic study including the photosensitizers of pyropheophorbide methyl ester (PPME) and pyropheophorbide a ( $\mathrm{PPa})$ was performed to study their photodynamic activity. The investigated photosensitizers in a homogeneous system of dimethylformamide (DMF) are not photostable upon irradiation. The photobleaching efficiency of $\mathrm{PPa}$ is higher than that of PPME. Combining these results with the data obtained by measuring the singlet oxygen quantum yield and the hydroxyl group generation, it was revealed that the photobleaching efficiency could be correlated with the singlet oxygen quantum yield and the hydroxyl group production of the photosensitizer.
\end{abstract}

Key words: Tumor — Singlet molecular oxygen — Free radicals — PPME — Fluorescence — Photobleaching - Absorption

\section{Introduction}

Upon light illumination many photosensitizers applied in biomedical studies are degraded. This process, usually called photobleaching, includes a decrease in the absorption and fluorescence. Studies on the photobleaching of hematoporphyrin-like compounds, as well as on certain chlorins and phthalocyanines have been performed in vitro and in vivo (Spiker and Bommer 1991; Jonig et al. 1993; Streckyte and Rotomskis 1993). In these studies, it was shown that the different photosensitizers photobleach at different rates in solutions, cells, and tissues. In tumors, it has also been observed the photobleaching of Photofrin II in patients undergoing photosensitized tumor therapy. Visible-absorbing photoproducts on illumination of hematoporphyrin or its derivatives were observed by some researchers (Mang et al. 1987; Moan and Kessel 1988; Jonig et al. 1993).

Some photosensitizers, in the process of photobleaching, are probably attacked by the singlet oxygen ${ }^{1} \mathrm{O}_{2}\left({ }^{1} \Sigma^{-}\right.$g $)$they produce, although free radicals may also play a role (Feix and Kalyanaraman 1991; Baker and Kanofsky 1992). This compilation is hoped that will help establishing the impor-

Correspondence to: Saleh Al-Omari, Department of Physics, The Hashemite University, Zarqa 13115, Jordan

E-mail: salomar@hu.edu.jo tance of singlet oxygen reactions in the phoptodegradation of dyes, pigments, polymer, as well as in harmful and/or beneficial photo-oxidations in biological system (Kanofsky 1991; Hoebeke and Damoiseau 2002).

In principle, the photobleaching of sensitizers used in the treatment of the solid tumors can be either an advantage or a disadvantage. During illumination, when the sensitizers are bleached too fast, the cancerous cells may not be completely destroyed (Moan 1986). On the other side, the photobleaching of the sensitizer can result in reducing its concentration in the target tissue, especially in the skin, and decreases the level of irreversible damage to normal tissue (Boyle and Potter 1987). Moreover, the photobleaching affects the photodynamic therapy (PDT) clinical dosimetry, since the initial concentration of the sensitizer in tissues decreases upon illumination (Potter et al. 1987).

In this study, attributed to the fact that the sensitizers exhibit different photobleaching behaviours in solutions, we have investigated the physical and chemical bleaching characteristics of pyropheophorbide methyl ester (PPME) and pyropheophorbide a $(\mathrm{PPa})$ in dimethylformamide (DMF) solvent. Based on these investigations, we have explored the phoptodegradation dependency on the singlet oxygen generated by the investigated molecules as well as on the formed radicals. This could help us to find the way how to match the photodegradation kinetics to the type of cancer in order to destruct it. 


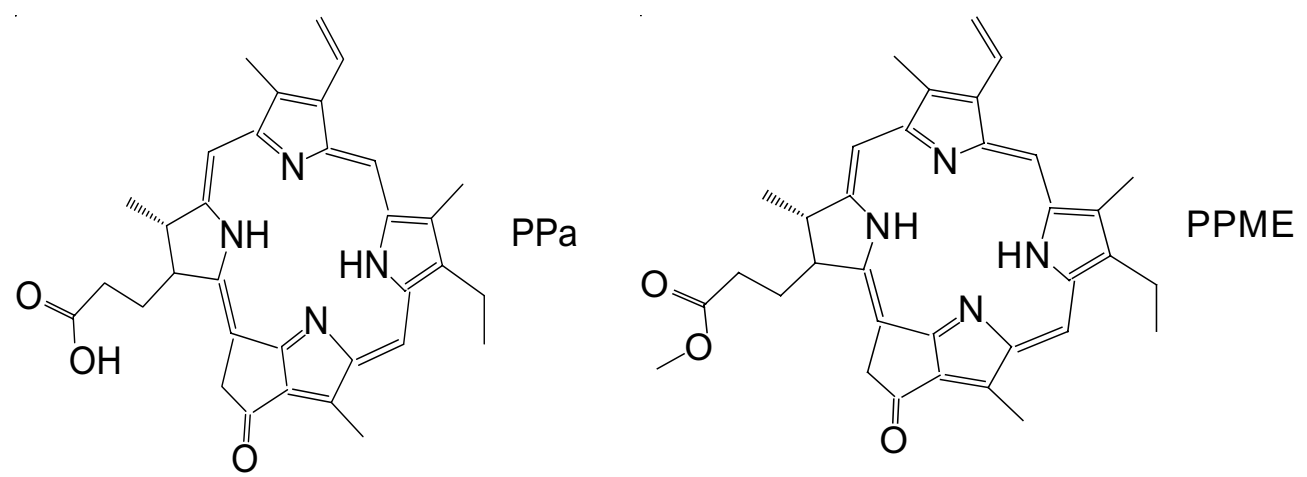

Figure 1. The structural formulas of pyropheophorbide a (PPa) and pyropheophorbide methyl ester (PPME).

\section{Materials and Methods}

\section{Materials}

9,10 dimethylanthracene (DMA) and PPME (95\% purity) were purchased from Sigma-Aldrich. The compounds were used as received without further purification and kept in the dark at low temperature of $-10^{\circ} \mathrm{C}$. $\mathrm{PPa}$ was taken as the reference compound. The structural formulas of $\mathrm{PPa}$ and PPME are shown in Fig. 1.

\section{Absorption spectroscopy}

The electronic ground-state absorption spectra were determined using a commercial spectrophotometer Shimadzu UV-1700 employing quartz cells $(0.01 \times 0.01 \mathrm{~m}$ path length, $l$ ) at room temperature of $25^{\circ} \mathrm{C}$. The increment step of the measurement was $0.1 \mathrm{~nm}$. In order to evaluate the molar extinction coefficients $(\varepsilon)$ of the investigated compounds, it was necessary to start from Eq. (5). The optical density $(O D)$ is plotted versus the concentration at a definite wavelength. Then, the fit is a linear regression with a slope of $\varepsilon l$. Because the cell length $l=0.01 \mathrm{~m}, \varepsilon$ can be obtained. The values of the other $\varepsilon$ values can be calculated by calibration with respect to the obtained one.

\section{Steady-state fluorescence spectroscopy}

Using an fluorescence spectrofluorimeter (model FS-900 CDT; Edinburgh Analytical Instruments, UK), the steadystate fluorescence spectra were collected employing fluorescence quartz cell $(0.01 \times 0.01 \mathrm{~m}$ path length $)$ at $25^{\circ} \mathrm{C}$. The investigated compounds were measured in $0.01 \times 0.01 \mathrm{~m}$ quartz optical cells using a combination of a cw-Xenon lamp, an emission monochromator (bandwidth 0.01-9 nm), and a polychromator with a cooled photomultiplier (model IP-28, Hamamastu). The width of the monochromator slit was fixed to $50 \mu \mathrm{m}$ during spectral measurements, enabling a spectral resolution of $0.05 \mathrm{~nm}$ and a maximum intensity of less than 20,000 counts corresponding to one-third of the photomultiplier saturation limit.

\section{Photobleaching measurements}

Phototransformation was produced by illumination $2.5 \mathrm{ml}$ samples placed in a $0.01 \times 0.01 \mathrm{~m}$ quartz cell using $\mathrm{He}-\mathrm{Ne}$ laser (emission wavelength: $632 \mathrm{~nm}$; light irradiance: 230 $\mathrm{Wm}^{-2}$ ). A water path was used to reduce the heat effect. The steady-state fluorescence was detected in perpendicular to the direction of excitation in an L-shaped setup. The concentration was set to be $10 \mu \mathrm{mol} / \mathrm{l}$ at the excitation wavelength of $632 \mathrm{~nm}$ for all samples of PPME and PPa. To observe the photodestruction, the steady state fluorescence spectra were frequently recorded for $7800 \mathrm{~s}$. The absorption spectra were registered before and after illumination.

For a photosensitizer with concentration $[\mathrm{m}]$ undergoes several excited state deactivation processes upon continuous stationary irradiation we can write

$$
\begin{aligned}
& \frac{\mathrm{d}[m]}{\mathrm{d} t}=-K[m] \\
& \ln [m]=\ln [m]_{0}-K \times t
\end{aligned}
$$

With

$$
\begin{aligned}
& K=I(t) \times K_{\mathrm{Fl}} \\
& \frac{\mathrm{d}[m]}{\mathrm{d} t}=-I(t) \times K_{\mathrm{Fl}}[m]
\end{aligned}
$$

Also we have

$$
O D=\varepsilon[m] l
$$

where $K\left(K^{\mathrm{PPME}}\right.$ or $\left.K^{\mathrm{PPa}}\right)$ refers to the rate constant of the photosensitizer deactivation, $I(t)$ is the light intensity absorbed by 


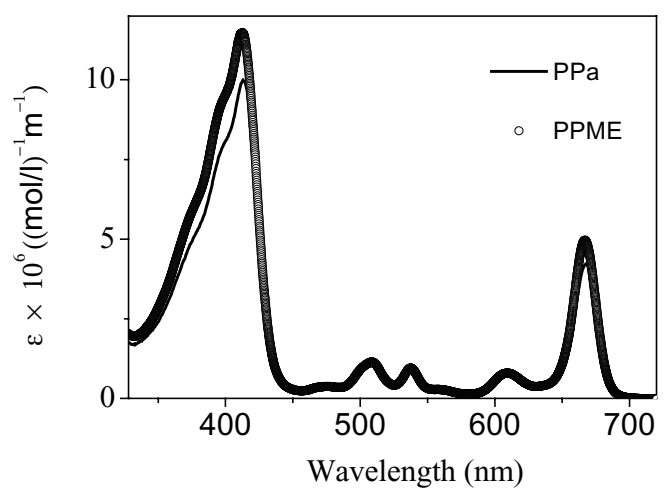

Figure 2. Electronic absorption spectra of $\mathrm{PPa}$ and PPME in DMF at room temperature of $25^{\circ} \mathrm{C}$.

the photosensitizer, and $K_{\mathrm{Fl}}$ is the initial fluorescence bleaching rate constant of the investigated sensitizer. Depending on Eq. (5), the concentration $[m]$ in the above equations can be replaced by the $O D$. In order to handle easily the calculation of the photobleaching rate constant we consider the aforementioned statements with the following argument:

As well-known, equal concentrations of photosensitizers have different optical densities at the illumination wavelength. This fact was taken into account when their photobleaching efficiencies were compared. The bleaching of the fluorescence emission for a sensitizer differs in magnitude corresponding to the time of illumination. To eliminate this dependency, it is used the relative intensity of the fluorescence bleaching values obtained by dividing the change in the fluorescence signal intensities $(\Delta F(t))$ by the value of the initial fluorescence $\left(F_{0}\right)$ before illumination. The fluorescence photobleaching of a photosensitizer is strongly related to the incident illumination dose $D$ which is the multiplication of the incident light fluence rate $I(t)$ and the irradiation time $t$. At the illumination wavelength, the absorbance of the photosensitizer defines the absorption of the radiation. Combining these parameters together with those above cited, $K_{\mathrm{Fl}}$ of the compounds under investigation can be evaluated by the following formula

$$
\frac{\Delta F(t)}{F_{0}}=-K_{\mathrm{Fl}} \times D \times O D_{\mathrm{exc}}
$$

where, $F(t)$ is the integrated area under the fluorescence spectrum at an illumination time of $t, F_{0}$ is the integrated area under the fluorescence spectrum at an illumination time of zero, $D=I(t) t$, and $O D_{\text {exc }}$ is the $O D$ at the excitation wavelength.

\section{Singlet oxygen measurements}

DMA with concentration of $0.5 \mathrm{mmol} / \mathrm{l}$ and absorbance at wavelength of $400 \mathrm{~nm}$, which is specific singlet oxygen-scav- enging agent, was followed by absorption spectroscopy to calculate the generation of the singlet oxygen $\left({ }^{1} \mathrm{O}_{2}\right)$ produced by the triplet excited state of PPME.

The absolute value of the singlet oxygen quantum yield of $\operatorname{PPME}\left(\Phi_{\Delta}{ }^{\mathrm{PPME}}\right)$ in a homogeneous medium of DMF was estimated using $\mathrm{PPa}$ as a standard $\left(\Phi_{\Delta}{ }^{\mathrm{PPa}}\right.$ is 0.52 in DMF) (Ali 2008). In the presence of DMA, ${ }^{1} \mathrm{O}_{2}$ is generated by the triplet state of the photosensitizer undergoes several decay processes upon continuous stationary irradiation. Therefore, Eq. (2) is valid and $[\mathrm{m}]$ accounts for the concentration of DMA. The bleaching constant $K$ now is the rate at which DMA concentration (or its absorbance) is consumed by ${ }^{1} \mathrm{O}_{2}$. Thus, we can write

$$
K=\left(1-10^{O D}\right) I_{\mathrm{exc}} \times \Phi_{\Delta} \times K_{\Delta}
$$

where $\left(1-10^{O D}\right) I_{\text {exc }}$ stands for the total absorbed photons by PPME or PPa at the excitation wavelength, $I_{\text {exc }}$ is identical for PPME and $\mathrm{PPa}$, and $K_{\Delta}$ is the total deactivation processes (physical and chemical) of ${ }^{1} \mathrm{O}_{2}$. For the same set up, $K_{\triangle}$ should be the same for PPME and PPa due to the similar conditions. Hence, $\Phi_{\Delta}{ }^{\text {PPME }}$ can be obtained by rearrangement Eq. (7):

$$
\Phi_{\Delta}^{\mathrm{PPME}}=\Phi_{\Delta}^{\mathrm{PPa}} \frac{\left(1-10^{O D^{\mathrm{PPa}}}\right)}{\left(1-10^{O D^{\mathrm{PPME}}}\right)} \frac{K^{\mathrm{PPME}}}{K^{\mathrm{PPa}}}
$$

where $O D^{\mathrm{PPa}}$ and $O D^{\mathrm{PPME}}$ are the absorbance of $\mathrm{PPa}$ and PPME, respectively. According to Eq. (2), $K^{\mathrm{PPa}}$ and $K^{\mathrm{PPME}}$ are the slopes kinetics of DMA disappearance photosensitized by PPa and PPME, respectively.

\section{Results}

\section{Absorption}

The electronic absorption spectra of PPa and PPME in DMF are shown in Fig. 2. As clear, the shape of the absorption spectrum of PPME resembles that of PPa with two strong vibration bands centered at $412.5 \mathrm{~nm}$ (belongs to Soret bands) with $\varepsilon_{412.5 \mathrm{~nm}}=1.15 \times 10^{7}(\mathrm{~mol} / \mathrm{l})^{-1} \mathrm{~m}^{-1}$ and at $666.8 \mathrm{~nm}$ (belongs to $\mathrm{Q}$ bands) with $\varepsilon_{666.8 \mathrm{~nm}}=4.96 \times 10^{6}(\mathrm{~mol} / \mathrm{l})^{-1} \mathrm{~m}^{-1}$. The other three maxima of $\mathrm{Q}$ bands are weaker with peaks positioned at 508,537 , and $609 \mathrm{~nm}$. Solutions of PPME in DMF followed the Beer-Lambert law up to $46 \mu \mathrm{mol} / \mathrm{l}$ indicating that under such conditions PPME is monomeric.

\section{Fluorescence}

The fluorescence spectra of PPa and PPME are shown in Fig. 3. Although the fluorescence spectra of both molecules 
have similar shapes, for PPME the maximum peak $\left(\lambda_{\max }=\right.$ $675 \mathrm{~nm}$ ) of the fluorescence spectrum is blue-shifted with respect to that of $\mathrm{PPa}\left(\lambda_{\max }=676 \mathrm{~nm}\right)$ (Fig. 3 and Table 1$)$. The estimations have shown that the fluorescence quantum yield $\left(\Phi_{\mathrm{F}}\right)$ is reduced for PPME to 0.25 compared to that of PPa (Table 1).

\section{Singlet oxygen generation}

$\Phi_{\Delta}{ }^{\text {PPME }}$ was evaluated in DMF. The used singlet oxygenscavenger was DMA in DMF. The kinetics of the DMA bleaching is first order linear regression fitting of the experimental data (Fig. 4). $\Phi_{\Delta}{ }^{\text {PPME }}$ was obtained by comparing the slope of DMA in the presence of PPME to the slope in the presence of PPa. Applying Eq. (8), $\Phi_{\Delta}{ }^{\mathrm{PPME}}$ was calculated to be 0.19 with a relative error of $3 \%$.

\section{Photobleaching kinetics}

Upon illumination PPa and PPME in DMF solvent a reduction in the absorption and fluorescence throughout the spectra occurs with no new band appearance or band shift over the whole spectral regions. The reduction has a linear character with increasing the illumination time. After $7800 \mathrm{~s}$ of illumination, the intensity, the spectral position, and the shape of the vibronic bands were the same. The kinetics of the bleaching and the bleaching rate constants are presented in Fig. 5 and Table 1, respectively. The photobleaching efficiency of $\mathrm{PPa}$ is stronger than that of PPME.

In order to look for the reason of having higher photobleaching rate constant for PPa and PPME compared to their free-mannitol compounds, it was investigated the effect of the mannitol quencher on the hydroxyl radical $\left(\mathrm{OH}^{*}\right)$. The findings exhibited a decrease in the bleaching efficiency by 0.11 for PPa and 0.03 for PPME in the presence of mannitol.

Table 1. Photophysical parameters of pyropheophorbide a (PPa) and pyropheophorbide methyl ester (PPME) in DMF at room temperature $\left(25^{\circ} \mathrm{C}\right)$

\begin{tabular}{|l|c|c|}
\hline & PPa & PPME \\
\hline$\lambda_{\max }{ }^{\mathrm{a}}(\mathrm{nm})$ & 667.5 & 666.8 \\
\hline$\lambda_{\max }{ }^{\mathrm{b}}(\mathrm{nm})$ & 676 & 675 \\
\hline$\Phi_{\Delta} \pm 0.03$ & 0.52 & 0.19 \\
\hline$\Phi_{\Delta}{ }^{\mathrm{PPa}} / \Phi_{\Delta}{ }^{\mathrm{PPME}}$ & \multicolumn{2}{|c|}{2.7} \\
\hline$\Phi_{\mathrm{F}} \pm 0.01$ & 0.31 & 0.07 \\
\hline $\mathrm{K}_{\mathrm{F} 1}\left(\times 10^{-7} \mathrm{~m}^{2} \cdot \mathrm{J}^{-1}\right)$ & 14 & 5 \\
\hline $\mathrm{K}_{\mathrm{F} 1}{ }^{\mathrm{PPa}} / \mathrm{K}_{\mathrm{F} 1}{ }^{\mathrm{PPME}}$ & \multicolumn{2}{|c|}{2.8} \\
\hline
\end{tabular}

$\Phi_{\Delta}$, singlet oxygen quantum yields; $\Phi_{\mathrm{F}}$, fluorescence quantum yields; $K_{\mathrm{F} 1}$, fluorescence bleaching rate constants; ${ }^{\mathrm{a}}$ peak maxima of the $\mathrm{Q}$ band absorption; ${ }^{\mathrm{b}}$ fluorescence maxima.

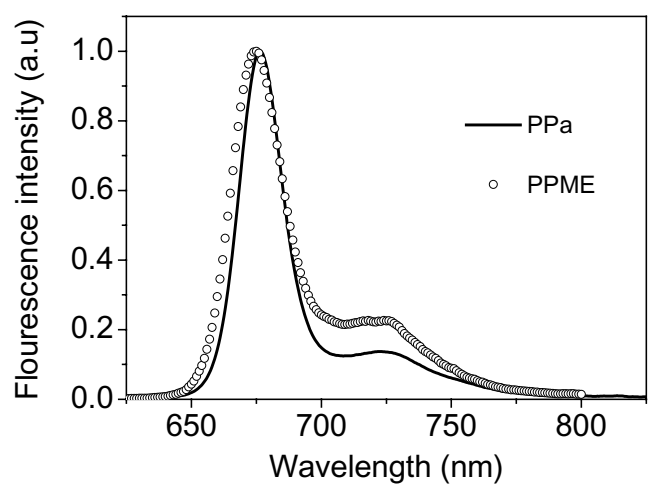

Figure 3. Fluorescence emission spectra of $\mathrm{PPa}$ and PPME in DMF at room temperature $\left(25^{\circ} \mathrm{C}\right)$. Excitation wavelength $\left(\lambda_{\max }\right)=412 \mathrm{~nm}$. Concentrations: $1.7 \mu \mathrm{mol} / \mathrm{l}$ PPME and $2 \mu \mathrm{mol} / \mathrm{l}$ $\mathrm{PPa}$.

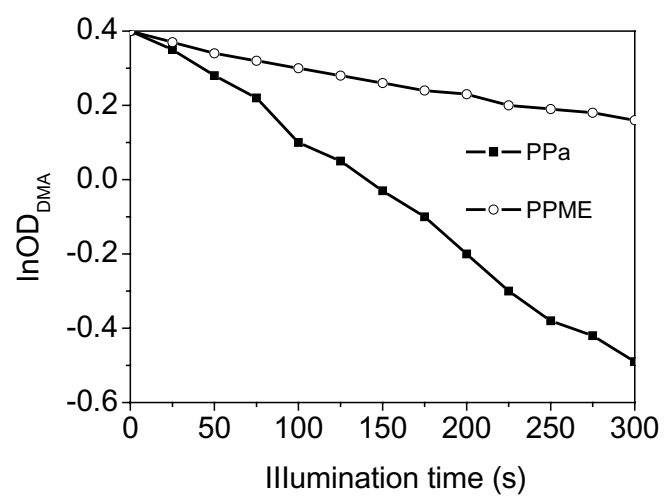

Figure 4. Dimethylanthracene (DMA) absorbance at $400 \mathrm{~nm}$ in the presence of the photoexcited of PPa and PPME in DMF at room temperature $\left(25^{\circ} \mathrm{C}\right) . O D_{\mathrm{DMA}}$ is the optical density of DMA. Concentration: $0.5 \mathrm{mmol} / \mathrm{l} \mathrm{DMA}$.

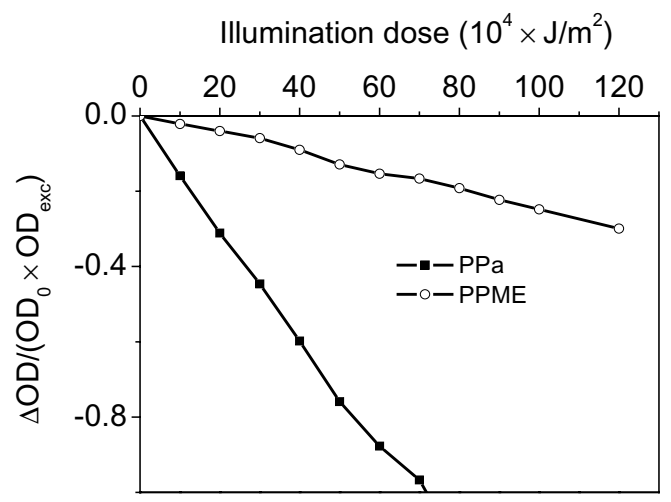

Figure 5. The kinetics of the fluorescence bleaching of PPa and PPME in DMF at room temperature $\left(25^{\circ} \mathrm{C}\right) . O D_{0}$, optical density at an illumination time of zero; $O D_{\text {exc }}$, optical density at the excitation wavelength. 


\section{Discussion}

PPME is a therapeutic second generation photosensitizer from the point of view of its potential application in the PDT of cancer (Matroule et al. 2001; Sun and Leung 2002). The photosensitizing activity is reduced attributed to the missing metal in the center of the PPME molecule (Spiker and Bommer 1991). Generally speaking, as compared with porphyrins PPa derivatives display vibronic absorption bands at longer wavelengths and exhibit higher molar extinction coefficients (Fig. 2 and Table 1) (Lin 1991). Moreover, such excellent spectroscopic properties are of great importance as they give higher efficiency of light absorption and subsequent photosensitization.

During irradiation, the fluorescence quantum yields of the investigated molecules are reduced after $7800 \mathrm{~s}$. The absorbance or fluorescence decreases monotonically with increasing the illumination time. The fluorescence quantum yields were 12 and 30\% less than their initial values for $\mathrm{PPa}$ and PPME, respectively. No changes of the spectral position or shapes of the absorption or fluorescence bands were observed. It was revealed that no any recovery in the dark has been detected when recording the absorption spectra as well as the fluorescence spectra after two days. Moreover, the intensity and the spectral shape of the bands were the same. Based on this, the bleaching of molecules generated as a result of illumination.

The kinetics of the photobleaching and bleaching rate constants are presented in Fig. 5 and Table 1, respectively. The sequence of the photobleaching efficiencies is $K_{\mathrm{Fl}} \mathrm{PPa}>$ $K_{\mathrm{Fl}}{ }^{\mathrm{PPME}}$. For both compounds the singlet oxygen quantum yield has the same order. This may not be surprising, since the photostability of the molecular systems depends strongly on their intersystem crossing quantum yields and hence on their singlet oxygen quantum yields. Thus, it is proposed that the photobleaching of PPME may be caused by ${ }^{1} \mathrm{O}_{2}$ according to the interaction:

$$
\begin{aligned}
& \mathrm{PPME}+\mathrm{O}_{2}\left({ }^{3} \Sigma_{g}^{-}\right)+\mathrm{h} v \rightarrow \mathrm{PPME}^{*}+{ }^{1} \mathrm{O}_{2} \\
& { }^{1} \mathrm{O}_{2}+\text { PPME or PPME }
\end{aligned}
$$

where PPME ${ }^{*}$ refers to the excited state of PPME. Despite that free radicals may be involved (Bensasson et al. 1993; Hoebeke et al. 1993). In general, during PDT, the cancerous tissue is mainly destroyed by the reactive oxygen species where ${ }^{1} \mathrm{O}_{2}$ is considered as the most effective cytotoxic agent involved in PDT (Krasnovsky et al. 1990; Baker et al. 1992). In this context, it has been shown that singlet oxygen causes single-strand breaks in DNA and that the guanine is the nucleoside base from which oxidative products are derived (Kanony et al. 2003). It is important to establish, as with all substrates, that particular products is produced as a result of singlet oxygen reaction. However, the biological damage might be caused by other reactive oxygen species such as hydrogen peroxide, superoxide anion radical $\left(\mathrm{O}_{2}{ }^{-}\right)$, and $\mathrm{OH}^{\bullet}$ (Georgakoudi et al. 1997). Furthermore, these species can also be released by mitochondria as second messengers during the apoptosis process (Wilson et al. 1997; Kessel et al. 2000).

As well-documented, that the molecular mechanism of the photodynamic effect has been under investigation for a long time and a first scheme was given by Foote (1987). He suggested two types of photosensitization both occurring in the presence of molecular oxygen:

Type I. The photosensitizer interacts with molecular oxygen via electron transfer mechanism. As a result, $\mathrm{O}_{2}{ }^{-}$is formed and the photosensitizer is transformed to a cation. This reaction has a very low quantum yield, especially if it occurs in a nonpolar solvent (Austrat 1986). It may become relevant at very high substrate concentrations. The outcome is a decrease in number of the photosensitizers that can be reexcited (Austrat 1986).

Type II. It is the most important type in PDT. ${ }^{1} \mathrm{O}_{2}$ is generated via energy transfer between the sensitizer molecule in the triplet state and the molecular oxygen in the triplet state (Rodgers 1993). Consequently, the photosensitizer transfers to the ground state and can absorb another photon of light. Thus, the photosensitizer plays a role of a catalyst (Ochsner 1997). In general, type II is especially dominant at normal oxygen concentration.

Type III. The last third way of photosensitizing which occurs in the absence of molecular oxygen and includes a charge transfer was postulated by Laustiat (Derycke and de Witte 2004). In this mechanism, the electron transfer mechanism occurs between the fluorophore in the first excited singlet state or in the first excited triplet state and a molecule except molecular oxygen (Wessels et al. 1997; Zhang and Rodgers 1995). The outcome is free radicals.

In type I following electron transfer from the photosensitizer to molecular oxygen, radicals are generated which interact in further dark reactions with biomolecules. The most important process in this type is the formation of the $\mathrm{O}_{2}{ }^{-}$which is generated by the interaction of molecular oxygen in its triplet ground state $\left(\mathrm{O}_{2}\right)$ with the excited sensitizer in its first excited state $\left(\mathrm{PPME}^{*}:{ }^{1} \mathrm{PPME}^{*}\right.$ or $\left.{ }^{3} \mathrm{PPME}^{*}\right)$. As a consequence, a cation radical of PPME $\left(\mathrm{PPME}^{+}\right)$is formed. The probability of recombination of the ionic radicals is higher in solvents of low dielectric constants. Therefore, the lifetimes of radicals generated in biotissue may be increased attributed to the large amount of water they contain. That means that more efficient damage could be earned. On the other hand, radicals involving in PPME photobleaching might be more evident in lipophilic tissues like biomembranes (Dougherty et al. 1992; Granville et al. 1998). 
Consequently, possible pathways of phoptodegradation for PPME in DMF are developed:

$$
\begin{aligned}
& \mathrm{PPME}^{*}+\mathrm{O}_{2} \rightarrow \mathrm{PPME}^{+}+\mathrm{O}_{2}^{-} \\
& \mathrm{PPME}^{+}+\mathrm{O}_{2}^{-} \rightarrow \mathrm{PPME}+\mathrm{O}_{2} \text { or product } \\
& 2 \mathrm{O}_{2}^{-}+2 \mathrm{H}^{+} \rightarrow \mathrm{H}_{2} \mathrm{O}_{2}+\mathrm{O}_{2}
\end{aligned}
$$

This reaction may happen in lipophobic solutions and it is seldom (Derycke and Witte 2004). But, since the generation of a large amount of ${ }^{1} \mathrm{O}_{2}\left(\Phi_{\Delta}\right.$ in Table 1) could be ruled out under these conditions (Dougherty et al. 1992), it is possible to conclude that $\mathrm{O}_{2}{ }^{-}$might not be ignored in the photobleaching of PPa and PPME. In addition, it might increase the oxidation of PPME more than PPa where the singlet oxygen generation is low (Table 1).

However, even the generation of ${ }^{1} \mathrm{O}_{2}$ could also enhance the production of the superoxide according to the following pathway

$$
\begin{gathered}
{ }^{1} \mathrm{O}_{2}+\mathrm{PPME}^{*} \leftarrow{ }^{1}\left[\mathrm{PPME}^{\delta+} \cdots \mathrm{O}_{2}^{\delta-}\right] \leftarrow \rightarrow \\
{ }^{3}\left[\mathrm{PPME}^{\delta+} \cdots \mathrm{O}_{2}^{\delta-}\right] \rightarrow{ }^{1} \mathrm{PPME}+\mathrm{O}_{2}^{-}
\end{gathered}
$$

If the charge-separated complexes shown above are able to dissociate to give separated radicals, this forms chemical quenching that can produce $\mathrm{O}_{2}{ }^{-}$which has been demonstrated for highly dielectric solvents such as water and with molecules that have low ionization potentials (Manring and Foote 1982).

On the other hand, type I is a photosensitizer-substrate interaction which requires high substrate concentrations (otherwise photosensitizer-oxygen interactions (type II) dominates) (Foote 1976). For such conditions, charge transfer from the photosensitizer to substrate molecules followed by the reaction of the formed anion radical with molecular oxygen giving $\mathrm{O}_{2}{ }^{-}$may happen (Derycke and Witte 2004). Nevertheless, the compounds under investigation have few micromolar concentrations which do not provide the convenient conditions to produce considerable amounts of $\mathrm{O}_{2}{ }^{-}$. Instead of that, the generation of singlet oxygen should be dominant in such case.

Another evidence could be a reason for having the fluorescence bleaching is to assume the occurrence of type III which includes electron transfer from the first excited singlet or triplet state of the photosensitizer to the substrate molecules, so radicals are formed. This route should not be taken into account because, as cited above, the concentrations of the compounds are very few. If this is the case, type II is the most probable manner of occurring the photodestrucions for the current complexes. Singlet oxygen is very reactive species that can nearly interact with most materials. Thus, the photosensi- tizer PPa with the highest singlet oxygen quantum yield would have the highest photobleaching rate constant (Table 1). On the contrary, PPME molecule with the lowest singlet oxygen quantum yield would have the least photobleaching rate constant. The photostability of molecular systems strongly depends on their singlet oxygen quantum yields (Fiedor et al. 2002). The measurements have shown that upon bubbling the samples, the photobleaching rates of the studied compounds become similar. This may enhance the assumption that photobleaching was mainly associated with singlet oxygen.

By observing the above reactions, it is demonstrated that regardless of being PPME in the ground state or in the excited state, it is susceptible to the attack by molecular oxygen and singlet oxygen. Such condition may cause a serious reduction of the unexpected low $\Phi_{\Delta}{ }^{\mathrm{PPME}}$ which is only 0.19 compared to the high value of $\Phi_{\Delta}{ }^{\mathrm{PPa}}$ which is 0.52 (Ali 2008). Another explanation could be a reason of having such a reduction of the singlet oxygen generation is that the absorption spectrum of PPa has a red shift in comparison with that of PPME (Fig. 2). This results in decreasing the energy gap between the first excited triplet state of the fluorophore and the singlet state of the molecular oxygen. Thus, more efficient energy transfer which should occur between them leads necessary to increase the singlet oxygen generation.

To support the above arguments, the mannitol which is able to quench $\mathrm{OH}^{*}$ (Feix and Kalyanaraman 1991) was employed. It showed a decrease in the photobleaching efficiency of the studied compounds. This points out that $\mathrm{OH}^{\circ}$ may be partially responsible for the photobleaching of these compounds. Because the decrease in the bleaching efficiency when using mannitol was 0.03 for PPME, this indicates that hydroxyl groups may play an important role in photodestruction of PPME. On the other hand, the presence of such radicals would play a role of damage when applying to cancerous cells. This could happen throughout two pathways: the first, because the studied molecules have $-\mathrm{COOCH}_{3}$ (carbomethoxy) group, it is susceptible to be attacked by ${ }^{1} \mathrm{O}_{2}$ producing $\mathrm{OH}^{*}$. The proposed mechanism of $\mathrm{OH}^{*}$ generation from PPME is according to the interaction:

$$
\begin{aligned}
& \text { PPME }+{ }^{1} \mathrm{O}_{2} \rightarrow\left[\mathrm{PPME}-{ }^{1} \mathrm{O}_{2}\right] \rightarrow \\
& \left\lfloor\mathrm{PPME}-\mathrm{COCH}_{2}^{+}\right\rfloor+\mathrm{OH}^{\bullet}+\mathrm{O}_{2}^{-}
\end{aligned}
$$

As seen, such interaction contributes to the reduction of ${ }^{1} \mathrm{O}_{2}$ production resulting in a less photodynamic activity. The second route was developed as follows:

$$
\begin{aligned}
& \mathrm{PPME}+\mathrm{PPME}^{*} \rightarrow \mathrm{PPME}^{-}+\mathrm{PPME}^{+} \\
& \mathrm{PPME}^{+}+\mathrm{H}_{2} \mathrm{O} \rightarrow \mathrm{PPMEH}^{+}+\mathrm{OH}^{\bullet} \\
& \mathrm{PPME}+\mathrm{OH}^{\bullet} \rightarrow \text { product }
\end{aligned}
$$


Furthermore, it was reported that ${ }^{1} \mathrm{O}_{2}, \mathrm{OH}^{\bullet}$, and $\mathrm{O}_{2}{ }^{-}$ produced by the photoexcited PPME are involved in the necrosis of colon cancer cells (Matroule et al. 2001; Sun and Leung 2002). Additionally, in the case of PPME, other reactive oxygen species in addition to ${ }^{1} \mathrm{O}_{2}$ were responsible for cell death probably implicated in the apoptosis mechanism (Matroule et al. 2001). In the view of improving the efficiency of PDT, the reactive oxygen species like $\mathrm{OH}^{\circ}$ and ${ }^{1} \mathrm{O}_{2}$ are potentially useful for enhancing the therapeutic applications of PPME.

An interesting point is worth to be mentioned is that the singlet oxygen and hydroxyl group were the main reactive oxygen species which are responsible for photobleaching PPME and PPa. This conclusion is based on comparison the values of the photobleaching efficiencies for the two molecules with the magnitudes of their singlet oxygen quantum yields. Thus, $K_{\mathrm{Fl}}{ }^{\mathrm{PPa}}$ is about three times greater than $K_{\mathrm{Fl}}{ }^{\mathrm{PPME}}$ which is approximately the same ratio when their singlet oxygen are compared (Table 1). The slight difference between the two ratios may be attributed to other kinds of the reactive oxygen species which are probably generated by various routes for the investigated compounds.

As well-known, the value of the singlet oxygen quantum yield for most PPa derivatives is generally high (Ali 2008). However, the singlet oxygen generation of PPME does not follow this trend. As seen above, we have attempted to explore some reasons behind the unexpected low value of the singlet oxygen of PPME. The two pigments have the same macrocylce (see, Fig. 1) which should enforce the two molecules to have similar photophysical behaviours. This is enhanced by the close photophysical parameters that most PPa derivatives have (Ali 2008). The functional groups are expected to have a minor effect on the photophysical properties of the molecule in vitro. In contrast to this fact, the carbomethoxy group of PPME seems to play an important role in determining its value of singlet oxygen since PPa has not this functional group.

We have proved above that under the illumination, PPME dissociates to give hydroxyl group radicals resulting in decreasing its population of the first excited singlet state. As a consequence, a reduction in the singlet oxygen generation occurs. In turn, hydroxyl groups may also attack PPME in its excited triplet state leading to another singlet oxygen reduction. Therefore, photobleaching efficiency could be correlated with the singlet oxygen quantum yield and hydroxyl group production of the photosensitizer. The photobleaching of photosensitizes could be either advantage or disadvantage in cancer therapy. It depends for instance on the tumor type or localization and thus, it is of great importance to know mechanisms how individual photosensitizers are degraded. This could help us to find the way how to match the bleaching kinetics to the cancer type in order to destruct it.
Acknowledgements. The authors would like to thank The Deanship of Research and Graduate Studies of Hashemite University for the financial support.

\section{References}

Ali A. (2008): Photophysical properties of pyropheophorbide a derivatives as potential photosensitizers for cancer treatment (Thesis). The Hashemite University, Jordan

Austrat G. (1986): Molecular mechanisms of photosensitization. Photochem. Photobiol. 162, 963-970

Baker A., Kanofsky J. R. (1992): Quenching of singlet oxygen by biomolecules from L1210 leukemia cells. Photochem. Photobiol. 55, 523-528

Bensasson R. V., Land E. J., Truscott T. G. (1993): Excited States and Free Radicals in Biology and Medicine: Contributions from Flash Photolysis and Pulse Radiolysis. (1st edition), Oxford University Press, New York

Boyle D. G., Potter W. R. (1987): Photobleaching of Photofrin II as a means of eliminating skin photosensitivity. Photochem. Photobiol. 46, 997-1001

Derycke A., de Witte P. M. (2004): Liposomes for photodynamic therapy. Adv. Drug. Delivery Rev. 56, 17-30

Dougherty T. J., Henderson B. W., Schwaetz S., Winkelman J. W., Lipson R. L. (1992): In Historical Perspective in Photodynamic Therapy, Basic Principles and Clinical Applications. (2nd edition), Marcel Dekker Inc., New York

Feix J., Kalyanaraman J. B. (1991): Production of singlet oxygenderived hydroxyl radical adducts during merocyanine540 mediated photosensitization: analysis by ESR-spin trapping and HPLC with electrochemical detection. Arch. Biochem. Biophys. 291, 43-51

Fiedor J., Fiedor L., Kammhuber N., Scherz A., Scheer H. (2002): Photodynamics of the bacteriochlorophyll-carotenoid system. 2 . Influence of central metal, solvent and $\beta$-carotene on photobleaching of bacteriochlorophyll derivatives. Photochem. Photobiol. 76, 145-152

Foote C. S. (1976): Free Radicals in Biology. Academic Press, New York

Foote C. S. (1987): Type I and Type II Mechanisms of Photodynamic Action in Light-Activated Pesticides. American Chemical Society, Washington DC

Georgakoudi I., Nichols M. G., Foster T. H. (1997): The mechanism of Photofrin photobleaching and its consequences for photodynamic dosimetry. Photochem. Photobiol. 65, 135-144

Granville D. J., Carthy C. M., Jiang H., Shore G. C., McManus B. M., Hunt D. W. (1998): Rapid cytochrome $c$ release, activation of caspases 3, 6, 7 and 8 followed by Bap31 cleavage in HeLa cells treated with photodynamic therapy. FEBS Lett. 437, 5-10

Hoebeke M., Seret A., Piette J., Van de Vorst A. (1993): Destruction of steric acid nitroxyl radicals mediated by photoexcited merocyanine 540 in liposomal and micellar system. Biochemistry 32, 2730-2736

Hoebeke M., Damoiseau X. (2002): Determination of the singlet oxygen quantum yield of bacteriochlorin a: a compara- 
tive study in phosphate buffer and aqueous dispersion of dimiristoyl-L-a-phosphatidylcholine liposomes. Photochem. Photobiol. Sci. 12, 283-287

König K., Schneckenburger H., Rück A., Steiner R. (1993): In vivo photoproduct formation during PDT with ALA-induced endogenous porphyrins. J. Photochem. Photobiol. B, Biol. 18, 287-290

Kanofsky J. R. (1991): Quenching of singlet oxygen. Photochem. Photobiol. 53, 93-99

Kanony C., Fabiano-Tixier A.-S., Ravanat J.-L., Vicendo P., Paillous N. (2003): Photosensitization of DNA damage by a new cationic pyropheophorbide derivative: sequence-specific formation of a frank scission. Photochem. Photobiol. 77, 659-667

Kessel D., Luo Y., Mathieu P., Reiners J. J. Jr. (2000): Determinants of the apoptotic response to lysosomal photodamage. Photochem. Photobiol. 71, 196-200

Krasnovsky A. A. Jr., Neverov K. V., Egorov S. Yu., Roeder B., Lewald T. (1990): Photophysical studies of pheophorbide a and pheophytin a. Phosphorescence and photosensitized singlet oxygen luminescence. J. Photochem. Photobiol. B, Biol. 5, 245-254

Lin C. W. (1991): Photodynamic therapy of malignant tumorsrecent developments. Cancer Cell 3, 437-444

Mang T. S., Dougherty T. J., Potter W. R., Boyle D. O., Sommer S., Moan J. (1987) Photobleaching of porphyrins used in photodynamic therapy and implications for therapy. Photochem. Photobiol. 45, 501-506

Manring L. E., Foote C. S. (1982): Chemistry of singlet oxygen. One-electron oxidation of tetramethylphenylenediamine singlet oxygen. J. Phys. Chem. 86, 1257-1259

Matroule J. Y., Carthy C. M., Granville D. J., Jolois O., Hunt D. C., Piette J. (2001): Mechanism of colon cancer cell apoptosis mediated by pyropheophorbide-a methyl ester photosensitization. Oncogene 20, 4070-4084

Moan J. (1986): Effect of bleaching of porphyrin sensitizers during photodynamic therapy. Cancer Lett. 33, 45-53
Moan J., Kessel D. (1988): Photoproducts formed from Photofrin II in cells. J. Photochem. Photobiol. B, Biol. 23, 429-436

Ochsner M. (1997): Photophysical and photobiological processes in the photodynamic therapy of tumours. J. Photochem. Photobiol. B, Biol. 39, 1-18

Potter W. R., Mang T. S., Dougherty T. J. (1987): The theory of photodynamic therapy dosimetry: consequences of photodestruction of sensitizer. Photochem. Photobiol. 46, 96-101

Rodgers L. (1993): Laser flash photolysis studies of the contact complex between molecular oxygen and 1-methylnaphalen. J. Phys. Chem. 97, 5643-5648

Spiker J. D., Bommer J. C. (1991): Chlorophyll and Related Pigments as Photosensitizers in Biology and Medicine. CRC Press, Florida

Streckyte G., Rotomskis R. (1993): Phototransformation of porphyrins in aques and micellar media. J. Photochem. Photobiol. B, Biol. 18, 259-263

Sun X., Leung W. N. (2002): Photodynamic therapy with pyropheophorbide a methyl ester in human lung carcinoma cancer cell: efficacy, localization and apoptosis. Photochem. Photobiol. 75, 644-651

Wessels J. M., Foote C. S., Ford W. E., Rodgers M. A. J. (1997): Photooxidation of tryptophan: $\mathrm{O}_{2}\left({ }^{1} \Delta_{\mathrm{g}}\right)$ versus electrontransfer pathway. Photochem. Photobiol. 65, 96-102

Wilson B. C., Olivo M., Singh G. (1997): Subcellular localization of Photofrin and aminolevulinic acid and photodynamic cross-resistance in vitro in radiation-induced fibrosarcoma cells sensitive or resistant to Photofrin-mediated photodynamic therapy. Photochem. Photobiol. 65, 166-176

Zhang X. Y., Rodgers M. A. J. (1995): Energy and electron transfer reactions of the MLCT state of ruthenium tris (bipyridyl) with molecular oxygen: a laser flash photolysis study. J. Phys. Chem. 99, 12797-12803

Received: October 10, 2008

Final version accepted: November 19, 2008 